EUROPEAN JOURNAL OF PURE AND APPLIED MATHEMATICS

Vol. 12, No. 1, 2019, 194-207

ISSN 1307-5543 - www.ejpam.com

Published by New York Business Global

\title{
Value-at-Risk Modeling with Conditional Copulas in Euclidean Space Framework
}

\author{
Vini Yves Bernadin Loyara ${ }^{1}$, Diakarya Barro ${ }^{2, *}$ \\ ${ }^{1}$ ESPK, BP 174 Kaya, Burkina Faso \\ ${ }^{2}$ UFR-SEG, U.O2, 12 BP:417 Ouaga 12, Burkina Faso
}

\begin{abstract}
This paper aims to establish an analytic relation between a time-varying conditional copula and the value at risk modeled by the underlying. Specically, under the assumption that the space is euclidean we use scalar product to clarify a link between the conditional copula varying with time and norms. It is then established a new expression on the geometric yield.
\end{abstract}

2010 Mathematics Subject Classifications: 51A50, 91C15, 60G57, 60H30, $62 \mathrm{H} 00$

Key Words and Phrases: Copulas, Euclidean space, Scalar product, VaR

\section{Introduction}

Modeling the risk of portfolio is to highlight the different methods or protocols to minimize the loss of values of a portfolio. The use of multivariate copulas in this modeling, is a contemporary approach, to develop indicators for the evaluation of the dependence between the different assets of this portfolio.

In multivariate theory of probability a pioneer theorem (Sklar,1959). Abe SKlar showed that the copula function enables to capture and to piece together the univariate models

(Sklar's Theorem). Therefore, every $n$-dimensional continuous distribution $H$ can be canonically parameterized by its univariate marginal $H_{1} ; \ldots ; H_{n}$ using $[0,1]^{n}$ a copula $C$ defined on the unit cube $[0 ; 1]$, such as

$$
H\left(x_{1}, \ldots, x_{n}\right)=C\left[H\left(x_{1}\right), \ldots, H_{n}\left(x_{n}\right)\right] ; \text { with }\left(x_{1}, \ldots, x_{n}\right) \in \overline{\mathbb{R}}^{n}=[-\infty,+\infty]^{n} .
$$

Under additional assumptions, differentiating the formula (1) shows that the density function of the copula is equal to the ratio of the joint density $h$ of $H$ to the product of $\mathrm{n}$ marginal densities $h_{i}$ such as, for all $\left(u_{1}, \ldots, u_{n}\right) \in[0,1]^{n}$,

$$
c\left(u_{1}, \ldots, u_{n}\right)=\frac{\partial^{n} C\left(u_{1}, \ldots, u_{n}\right)}{\partial u_{1} \times \ldots \times \partial u_{n}}=\frac{h\left[H_{n}^{-1}\left(u_{1}\right), \ldots, H_{n}^{-1}\left(u_{n}\right)\right]}{h_{1}\left[H_{1}^{-1}\left(u_{1}\right) \times \ldots \times H_{n}^{-1}\left(u_{n}\right)\right]} .
$$

*Corresponding author.

DOI: https://doi.org/10.29020/nybg.ejpam.v12i1.3347

Email addresses: loyarayves@outlook.com (V. Y. B. Loyara), dbarro2@gmail.com (D. Barro) 
where $H_{i}^{-1}$ is the quantile function of $H_{i}$; that is, $H_{i}^{-1}(u)=\inf \left\{x \in \mathbb{R}, H\left(x_{i}\right) \geq u\right\}$. In stochastic financial analysis, from the definition in the univariate case we know that the quantile function provides a point that accumulates a probability for the left tail and for the right tail. The univariate quantile function $Q_{X}(\alpha)$ is used in the risk theory to define the univariate risk measure : the value at risk is defined.

More generally in multivariate study, for a random vector $X$ satisfying the regularity conditions, we define the multidimensional VaR at probability level $\alpha$ by :

$$
\operatorname{VaR}_{\alpha}(X)=\mathbb{E}[X \mid X \in \partial L(\alpha)]
$$

where $\partial L(\alpha)$ is the boundary of the $\alpha$ - level set of $F$, the univariate component of the vector : $\operatorname{VaR}_{\alpha}(X)$ are such as, for all portfolio $X_{i} ; \operatorname{VaR}_{\alpha}(X)=\left\{z / F_{X_{i}}(z) \geq \alpha\right\}=$ $F_{X_{i}}^{-1}(\alpha), F_{X_{i}}^{-1}$ being the right continuous inverse of $F_{X}$.

In this paper, it is matter of the notion of multivariate risk coupled with that of conditional copula varying with time. We have established, subject of being in a Euclidean space, a relation between the conditional copula varying with time and the scalar product or norm. We are inspired by Patton's work on conditional copulas varying with time. Indeed, from proposition and Sklar's theorem adapted to the conditional copula (which are all from Patton) we use the relation (5) to obtain our different results. Finally, a new expression on the geometric yield is established.

\section{Preliminaries}

In this section we have grouped together the different notion definitions, propositions and theorems which will be useful thereafter. We need Sklar's theorem and its adaptation in the conditional case proposed by Patton (2006) to elaborate the different results we found.

\subsection{A survey of Conditional Copulas}

In copulas theory Joe (1997) or Nelsen (2007) provide detailed and readable introductions to copulas and their statistical and mathematical foundations while Bouy et al. (2000) or Cherubini et al. (2004) deal with applications of copulas to different levels of financial issues and derivatives pricing.

A $n$-dimensional copula is a multivariate distribution function $C:[0,1]^{n} \longrightarrow[0,1]$ satisfying the following properties

i) Grounded: $C\left(u_{1}, \ldots, u_{i-1}, 0, u_{i+1}, \ldots, u_{n}\right)=0$ for all $i$ and all $\left(u_{1}, \ldots, u_{i-1}, u_{i+1}, \ldots, u_{n}\right) \in$ $[0,1]^{n-1}$.

ii) copula marginal : $C\left(u_{1}, \ldots, u_{i-1}, 1, u_{i+1}, \ldots, u_{n}\right)$ is an $(n-1)$ copula for all $i \in$ $\{1, \ldots, n\}$.

iii) $n$-increasingness : the volume $V_{B}$ of any rectangle $B=[a, b] \subseteq[0,1]^{n}$ is positive,

$$
\left.\left.V_{B}=\int_{B} d C\left(u_{1}, \ldots, u_{n}\right)=i_{1}=1\right] 2 \sum \ldots i_{n}=1\right] 2 \sum(-1)^{i_{1}+\ldots+i_{n}} C\left(u_{i_{1}}, \ldots, u_{i_{n}}\right) \geq 0 .
$$


Using the above relation (4) (positiveness of the volume of any hyper-rectangle of $\overline{\mathbb{R}}^{n}$ ) Barro et all.(2012) provide the following result by extending a proposition of Patton (2002) both to space-varying case and to higher dimensional framework.

Proposition 1. Let $F_{t, w_{t}}$ denote the joint distribution of $\left(\tilde{X}_{t, n-1}, W_{t}\right), t \in T$ with $\tilde{X}_{t, n-1}=\left(X_{t, 1}, \ldots, X_{t, n-1}\right)$, then the conditional time-varying distribution of $\left(\tilde{X}_{t, n-1}, W_{t}\right)$ is given, for all $\tilde{y}_{t} \in(\overline{\mathbb{R}})^{n-1}$ by

$$
H_{t, w_{t}}\left(x_{t} / w_{t}\right)=f_{w}^{-1}\left(w_{t}\right) \frac{\partial H_{t, w_{t}}\left(x_{t, 1}, \ldots, x_{t, n-1}, w_{t}\right)}{\partial w_{t}}
$$

where $f_{w}$ is the spacial density of the law of $W_{t}$. Moreover, the following properties are satisfied

(i) $H_{t, w_{t}}\left(x_{t, 1}, \ldots,-\infty, \ldots, x_{t, n-1}, w_{t}\right)=0$ for all $\tilde{y}_{t} \in(\overline{\mathbb{R}})^{n-1}$.

(ii) $H\left(\infty, \ldots, \infty / w_{t}\right)=1$ for all $\tilde{y}_{t} \in(\overline{\mathbb{R}})^{n-1}$.

(iii) For all $\tilde{x}_{t}^{(1)}=\left(x_{t, 1}^{(1)}, \ldots, x_{t, n-1}^{(1)}\right) \in(\overline{\mathbb{R}})^{n-1}$ and $\tilde{y}_{t}^{(1)}=\left(x_{t, 1}^{(2)}, \ldots, x_{t, n-1}^{(2)}\right) \in(\overline{\mathbb{R}})^{n-1}$ such as $x_{t, j}^{(1)} \leq x_{t, j}^{(2)}$ then

$$
\sum_{\left(i_{1}, \ldots, i_{n}\right) \in\{1,2\}^{n}}(-1)^{\left.\mathfrak{j}^{j}=1\right] n \sum j_{j}} H_{n-1, w_{t}}\left(x_{t, 1}^{\left(i_{1}\right)}, \ldots, x_{t, n-1}^{\left(i_{n-1}\right)}, w_{t}\right) \geq 0 .
$$

Let's consider a linear portfolio of consisting of $n$ different financial instruments (risks, actions) $X=\left(X_{1}, \ldots, X_{n}\right)$. Further, let $p_{0}=\left(p_{0,1}, \ldots, p_{0, n}\right)$ the initial value of the portfolio is given by $\left.V_{0}=i=1\right] n \sum x_{i} p_{0, i}$ for a realization $x=\left(x_{1}, \ldots, x_{n}\right)$ of $X$. At the next date $t$ the uncertain Profit and Loss function of the portfolio is given by

$$
\left.F_{t}\left(x_{1}, \ldots, x_{n}\right)={ }_{i=1}^{[}\right]_{n} \sum x_{i}\left(p_{0, i}-p_{t, i}\right)=i[1] n \sum x_{i} p_{t, i}\left(e^{z_{t, i}}-1\right) .
$$

where $Z_{t}=\left(z_{t, i}, \ldots, z_{t, n}\right)$ is the $\log$ Price vector such as $z_{t, i}=\log p_{t, i}$. Particularly, from the integral probability transforms we can associate to $F_{t}$, a parametric copula $C_{t}$ such as, for all $\left(u_{t, 1}, \ldots, u_{t, n}\right) \in[0,1]^{n}$,

$$
C_{t}\left(u_{t, 1}, \ldots, u_{t, n}\right)=P\left(F_{t, 1}\left(X_{1}\right) \leq u_{t, 1}, \ldots, F_{t, n}\left(X_{n}\right) \leq u_{t, n}\right)
$$

\subsection{Scalar product and copulas application on VaR}

According to Karl Friedrich Siburg et al. (1975), the restrictions of $\langle\rangle,\|\|$, and $d$ to $\mathfrak{C}_{n}$ are called the sobolev scalar product, the Sobolev norm and the Sobolev distance function on $\mathfrak{C}_{n}$, respectively. But we suppose a new norm in a space a Euclidean vector space is a prehilbert space of finite dimension. It is complete. 
Definition 1. Consider $E=\mathbb{R}^{n}$ a vector space with the scalar product $\langle$,$\rangle . In the following$ we consider ourselves in finite dimension and $(E,\langle\rangle$,$) is Euclidean space. The application$

$$
x \longmapsto\|x\|=\sqrt{\langle x, x\rangle}
$$

defines on $E$ a norm, called euclidean norm and noted \|\|$. \forall(x, y) \in E^{2}$ we have :

- Cauchy-Schwartz inequality :

$$
|\langle x, y\rangle| \leq\|x\|\|y\|
$$

- Cauchy-Schwartz equality (in the case where $(x, y)$ do not form a free family):

$$
|\langle x, y\rangle|=\|x\|\|y\|
$$

- Polarization identity :

$$
\langle x, y\rangle=\frac{1}{4}\left(\|x+y\|^{2}+\|x-y\|^{2}\right)
$$

In Euclidean space there is an orthogonal basis and the Gram-Schimdt process allows to build it. In a Euclidean space, any orthogonal family can be completed in an orthogonal basis. Let an orthogonal base of a euclidean vector space $(E,\langle\rangle$,$) and u$ an endomorphism from E:

\section{Main Results}

Let's consider a linear portfolio of consisting of $n$ different financial instruments (risks, actions) $X=\left(X_{1}, \ldots, X_{n}\right)$ and let $p_{t}=\left(p_{1, t}, p_{2, t}, \ldots, p_{n, t}\right)$ at a given date measured at given time t. Further, let $p_{0}=\left(p_{0,1}, \ldots, p_{0, n}\right)$ the initial value of the portfolio is given by $\left.V_{0}=\stackrel{[}{[}=1\right] n \sum x_{i} p_{0, i}$ for a realization $x=\left(x_{1}, \ldots, x_{n}\right)$ of $X$.

$$
\left.p_{t}=\left(p_{1, t}, \ldots, p_{n, t}\right)=i=1\right] n \sum\left\langle p_{i, t}, e_{i}\right\rangle e_{i}, \text { and }\left\|p_{t}\right\|=\sqrt{{ }_{i=1] n} \sum p_{i, t}^{2}}=\sqrt{\left.{ }_{i=1}^{[}\right]_{n} \sum\left\langle p_{t}, e_{i}\right\rangle^{2}} \text {. }
$$

Consider

$$
\left.P_{t}=\left(p_{1, t} e^{z_{t, 1}}, \ldots, p_{n, t} e^{z_{n, 1}}\right)={ }_{i=1}^{[}\right]_{n} \sum\left\langle p_{i, t} e^{z_{t, i}}, e_{i}\right\rangle e_{i}
$$

and

$$
\left\|P_{t}\right\|=\sqrt{\left.{ }_{i=1}^{[}\right]_{n} \sum p_{i, t}^{2} e^{2 z_{t, i}}}=\sqrt{\left.{ }_{i=1}^{[}\right]_{n} \sum\left\langle P_{t}, e_{i}\right\rangle^{2}} .
$$

The concept of scalar product that allowed us to highlight a link between the conditional copula and the notion of norms in the metric spaces. Let assume that we are in an euclidean space and that all necessary conditions are fulfilled. We use the characteristic elements of metric spaces (euclidean space), to establish with the notion of scalar product or norm a relation between the VaR and the conditional time-dependent copula. 


\subsection{Scalar product and copulas applications on the VaR}

The following sub-section proposal was inspired by the Gram-Schmidt process. We think it is necessary to depend the proposition 2 afterwards.

Proposition 2. Let $E$ be a Euclidean space and $\left(e_{1}, \ldots, e_{n}\right)$ be a base of $E$ in which. Then, there is a only base $\xi=\left(\xi_{1}, \ldots, \xi_{n}\right)$ such that; if

$$
\left.\operatorname{VaR}_{u}(X)=\left(\operatorname{VaR}_{u_{1}}\left(X_{1}\right), \ldots, \operatorname{VaR}_{u_{n}}\left(X_{n}\right)\right)=i=1\right] n \sum\left\langle\operatorname{VaR}_{u_{i}}, e_{i}\right\rangle e_{i}
$$

then

$$
\left.\left\langle\operatorname{VaR}_{u}(X), \xi\right\rangle-{ }_{i=2}^{[}\right] n \sum \frac{V a R_{u_{i}} \vartheta_{i}}{\left\|\vartheta_{i}\right\|}=\operatorname{Va} R_{u_{1}} \frac{e_{1}}{\left\|e_{1}\right\|}
$$

with $\xi_{1}=\frac{e_{1}}{\left\|e_{1}\right\|} \quad$ and $\quad \forall i \in\{1, \ldots, n-1\}$, and

$$
\left.\xi_{i+1}=\frac{\vartheta_{i+1}}{\left\|\vartheta_{i+1}\right\|} \quad \text { with } \vartheta_{i+1}=e_{i+1}-k=1\right] i \sum\left\langle e_{i+1}, \xi_{k}\right\rangle \xi_{k}
$$

Proof. By assumption $E$ is a Euclidean space and let $\left(e_{1}, e_{2}, \ldots, e_{n}\right)$ be a base of $E$ such that

$$
\left.\operatorname{VaR}_{u}(X)=\left(\operatorname{VaR}_{u_{1}}\left(X_{1}\right), \ldots, \operatorname{VaR}_{u_{n}}\left(X_{n}\right)\right)=i=1\right]_{n} \sum\left\langle\operatorname{VaR}_{u_{i}}, e_{i}\right\rangle e_{i} .
$$

So, it follows that,

$$
\|\operatorname{VaR}(X)\|=\sqrt{i=1] n \sum V a R_{u_{i}}^{2}}=\sqrt{{ }_{i=1] n} \sum\left\langle\operatorname{VaR} R_{u}, e_{i}\right\rangle^{2}}
$$

The orthogonalization process of Gram-Schimdt (1875) allows us to say that there is only one base $\left(\xi_{1}, \ldots, \xi_{n}\right)$ as

$\xi_{1}=\frac{e_{1}}{\left\|e_{1}\right\|} \quad$ and $\quad \forall i \in\{1, \ldots, n-1\}, \xi_{i+1}=\frac{\vartheta_{i+1}}{\left\|\vartheta_{i+1}\right\|} \quad$ with $\left.\vartheta_{i+1}=e_{i+1}-k=1\right] i \sum\left\langle e_{i+1}, \xi_{k}\right\rangle \xi_{k}$.

Futhermore, it comes that :

$$
\begin{gathered}
\left.\left\langle V a R_{u}(X), \xi\right\rangle=i=1\right] n \sum V a R_{u_{i}} \xi_{i} \\
\left.\left\langle V a R_{u}(X), \xi\right\rangle-{ }_{i=2}^{[}\right] n \sum \frac{V a R_{u_{i}} \vartheta_{i}}{\left\|\vartheta_{i}\right\|}=V a R_{u_{1}} \frac{e_{1}}{\left\|e_{1}\right\|}
\end{gathered}
$$

Let's consider a linear portfolio of consisting of $n$ different financial instruments (risks, actions) $X=\left(X_{1}, \ldots, X_{n}\right)$ and let $p_{t}=\left(p_{1, t}, p_{2, t}, \ldots, p_{n, t}\right)$ at a given date measured at given time t. Further, let $p_{0}=\left(p_{0,1}, \ldots, p_{0, n}\right)$ the initial value of the portfolio is given by $\left.V_{0}=i=1\right] n \sum x_{i} p_{0, i}$. 
Theorem 1. For a realization $x=\left(x_{1}, \ldots, x_{n}\right)$ of $X$, at the next date the uncertain Profit and Loss function of the portfolio is given by

$$
\left.\left.F_{t}\left(x_{1}, \ldots, x_{n}\right)=i=1\right] n \sum x_{i}\left(p_{0, i}-p_{t, i}\right)=i=1\right] n \sum x_{i} p_{t, i}\left(e^{z_{t, i}}-1\right) ;
$$

then

$$
C_{t}\left(u_{1}, \ldots, u_{n}\right)=\|\operatorname{VaR}(X)\|\left\|P_{t}-p_{t}\right\|
$$

Furthermore,

$$
C_{t}\left(u_{1}, \ldots, u_{n}\right)=\frac{1}{4}\left(\left\|\operatorname{VaR} R_{u}(X)+P_{t}-p_{t}\right\|^{2}+\left\|\operatorname{VaR} R_{u}(X)-\left(P_{t}-p_{t}\right)\right\|^{2}\right)
$$

and where $P_{t}=\left(p_{1, t} e^{z_{t, 1}}, \ldots, p_{n, t} e^{z_{n, 1}}\right)$,

$$
\operatorname{VaR}_{u}(X)=\left(\operatorname{VaR}_{u_{1}}\left(X_{1}\right), \ldots, \operatorname{VaR}_{u_{n}}\left(X_{n}\right)\right) .
$$

is a Value at risk of the $X$ and $\|\cdot\|$ euclidean norm and noted.

Proof. Consider the following relation

$$
C_{t}\left(u_{1}, \ldots, u_{n}\right)=F_{W_{t}}\left(F_{1, W_{t}}^{(-1)}\left(u_{1}\right), \ldots, F_{n, W_{t}}^{(-1)}\left(u_{n}\right)\right) .
$$

Ff we consider relation (7) we obtain,

$$
\left.C_{t}\left(u_{1}, \ldots, u_{n}\right)=i=1\right] n \sum V a R_{u_{i}}\left(X_{i}\right)\left(p_{t, i} e^{z_{t, i}}-p_{t, i}\right) .
$$

Consider $P_{t}=\left(p_{1, t} e^{z_{t, 1}}, \ldots, p_{n, t} e^{z_{n, 1}}\right)$ and $\operatorname{VaR}_{u}(X)=\left(\operatorname{VaR}_{u_{1}}\left(X_{1}\right), \ldots, \operatorname{VaR}_{u_{n}}(X)\right)$. Then,

$$
C_{t}\left(u_{1}, \ldots, u_{n}\right)=\left\langle\operatorname{VaR}_{u}(X), P_{t}-p_{t}\right\rangle
$$

Value at risk is intrinsically linked to the portfolio and therefore to the initial amount and the amount at a given time t. We will suppose linked vector $\operatorname{VaR}_{u}(X)$ and vector $p_{t}-P_{t}$. The relation 10 we give

$$
\left|\left\langle\operatorname{VaR}_{u}(X), P_{t}-p_{t}\right\rangle\right|=\left\|\operatorname{VaR}_{u}(X)\right\|\left\|P_{t}-p_{t}\right\| .
$$

Then,

$$
C_{t}\left(u_{1}, \ldots, u_{n}\right)=\|\operatorname{VaR}(X)\|\left\|P_{t}-p_{t}\right\|
$$

and equality (11) given

$$
C_{t}\left(u_{1}, \ldots, u_{n}\right)=\frac{1}{4}\left(\left\|V a R_{u}(X)+P_{t}-p_{t}\right\|^{2}+\left\|V a R_{u}(X)-\left(P_{t}-p_{t}\right)\right\|^{2}\right)
$$

The following result allows us to obtain; 
Proposition 3. Let $p_{t}=\left(p_{1, t}, p_{2, t}, \ldots, p_{n, t}\right)$ at a given date measured at given time $t$. And suppose these risks represent potential losses in dependent lines of business for example an insurance company. Then

$$
C\left(u_{1}, \ldots, u_{n} / w_{t}\right)=f_{w}^{-1}\left(w_{t}\right)\left\langle\frac{\partial}{\partial w_{t}}\left(\operatorname{VaR}_{u}\left(X / w_{t}\right)\right),\left(P_{t}-p_{t}\right)\right\rangle
$$

and where $P_{t}=\left(p_{1, t} e^{z_{t, 1}}, \ldots, p_{n, t} e^{z_{n, 1}}\right), \operatorname{VaR} R_{u}\left(X / w_{t}\right)$ is a Value at risk of the $X$ such that $w_{t}$ and \|\| euclidean norm and noted.

Proof. Let $F_{W_{t}}$ be any conditional time-varying conditional distribution with marginal $\left\{F_{i, W_{t}} ; 1 \leq i \leq n\right\}$. Then there exists a only copula $C:[0,1]^{n} \longrightarrow[0,1]$ such as

$$
C\left(u_{1}, \ldots, u_{n} / w_{t}\right)=F_{W_{t}}\left(F_{1, W_{t}}^{(-1)}\left(u_{1} / w\right), \ldots, F_{n, W_{t}}^{(-1)}\left(u_{n} / w\right)\right)
$$

where $F_{i, W_{t}}^{(-1)}\left(u_{i} / w\right)=\inf \left\{x: F_{i, W_{t}}(x / w) \geq u_{i}\right\}$ for each $u_{i}$ and $w_{t} \in W_{t}$. Then, it follows that :

$$
C\left(u_{1}, \ldots, u_{n} / w_{t}\right)=\frac{\partial F_{W_{t}}\left(F_{1, W_{t}}^{(-1)}\left(u_{1} / w_{t}\right), \ldots, F_{n-1, W_{t}}^{(-1)}\left(u_{n-} / w_{t}\right), w_{t}\right)}{\partial w_{t}} .
$$

By considering the equality (7) int the following relation

$$
C\left(u_{1}, \ldots, u_{n} / w_{t}\right)=F_{W_{t}}\left(F_{1, W_{t}}^{(-1)}\left(u_{1} / w_{t}\right), \ldots, F_{n, W_{t}}^{(-1)}\left(u_{n} / w_{t}\right) / w_{t}\right)
$$

are obtains :

$$
\begin{aligned}
C\left(u_{1}, \ldots, u_{n} / w_{t}\right) & =f_{w}^{-1}\left(w_{t}\right) \times[i=1] n \sum \frac{\partial\left(\operatorname{VaR}_{u_{i}}\left(X_{i} / w_{t}\right)\right)}{\partial w_{t}} p_{i, t} e^{z_{t, i}} \\
& \left.-{ }_{i=1}[]_{n} \sum \frac{\partial\left(\operatorname{VaR}_{u_{i}}\left(X_{i} / w_{t}\right)\right)}{\partial w_{t}} p_{i, t}\right]
\end{aligned}
$$

Consider $P_{t}=\left(p_{1, t} e^{z_{t, 1}}, \ldots, p_{n, t} e^{z_{n, 1}}\right)$, we have :

$$
\begin{gathered}
C\left(u_{1}, \ldots, u_{n} / w_{t}\right)=f_{w}^{-1}\left(w_{t}\right) \times\left\langle\frac{\partial}{\partial w_{t}} \operatorname{VaR} R_{u}\left(X / w_{t}\right), P_{t}\right\rangle-f_{w}^{-1}\left(w_{t}\right) \times\left\langle\frac{\partial}{\partial w_{t}} \operatorname{VaR}\left(X / w_{t}\right), p_{t}\right\rangle \\
C\left(u_{1}, \ldots, u_{n} / w_{t}\right)=\left\langle\frac{\partial}{\partial w_{t}} \operatorname{VaR}\left(X / w_{t}\right), f_{w}^{-1}\left(w_{t}\right)\left(P_{t}-p_{t}\right)\right\rangle
\end{gathered}
$$

if $f_{w}^{-1} \in \mathcal{C}^{1}$ we can write, then

$$
C\left(u_{1}, \ldots, u_{n} / w_{t}\right)=f_{w}^{-1}\left(w_{t}\right)\left\langle\frac{\partial}{\partial w_{t}}\left(\operatorname{VaR}_{u}\left(X / w_{t}\right)\right),\left(P_{t}-p_{t}\right)\right\rangle
$$


Proposition 4. Let these risks represent potential losses in dependent lines of business for an insurance company for example. In the following we consider ourselves in finite dimension and $(E,\langle\rangle$,$) is Euclidean space. Further, the conditional copula of is given by$

$$
C\left(u_{1}, \ldots, u_{n} / w_{t}\right)=\left|f_{w}^{-1}\left(w_{t}\right)\right|\left\|\frac{\partial}{\partial w_{t}}\left(\operatorname{VaR}_{u}\left(X / w_{t}\right)\right)\right\|\left\|P_{t}-p_{t}\right\|
$$

Furthermore

$$
\begin{aligned}
C\left(u_{1}, \ldots, u_{n} / w_{t}\right) & =\frac{1}{4} f_{w}^{-1}\left(w_{t}\right)\left(\left\|\frac{\partial}{\partial w_{t}}\left(\operatorname{VaR}_{u}\left(X / w_{t}\right)\right)+\left(P_{t}-p_{t}\right)\right\|^{2}\right. \\
& \left.+\left\|\frac{\partial}{\partial w_{t}}\left(\operatorname{VaR}_{u}\left(X / w_{t}\right)\right)-\left(P_{t}-p_{t}\right)\right\|^{2}\right)
\end{aligned}
$$

Proof. For this proof, consider the following relation :

$$
C\left(u_{1}, \ldots, u_{n} / w_{t}\right)=f_{w}^{-1}\left(w_{t}\right)\left\langle\frac{\partial}{\partial w_{t}}\left(V a R_{u}\left(X / w_{t}\right)\right), P_{t}-p_{t}\right\rangle ;
$$

Moreover considering equality (10) it came that;

$$
\left|f_{w}^{-1}\left(w_{t}\right)\left\langle\frac{\partial}{\partial w_{t}}\left(\operatorname{VaR}_{u}\left(X / w_{t}\right)\right), P_{t}-p_{t}\right\rangle\right|=\left|f_{w}^{-1}\left(w_{t}\right)\right|\left\|\frac{\partial}{\partial w_{t}}\left(\operatorname{VaR} R_{u}\left(X / w_{t}\right)\right)\right\|\left\|P_{t}-p_{t}\right\|
$$

so

$$
C\left(u_{1}, \ldots, u_{n} / w_{t}\right)=\left|f_{w}^{-1}\left(w_{t}\right)\right|\left\|\frac{\partial}{\partial w_{t}}\left(\operatorname{VaR}_{u}\left(X / w_{t}\right)\right)\right\|\left\|P_{t}-p_{t}\right\|
$$

and if we take it the relation (11)

$$
\begin{aligned}
\left\langle\operatorname{VaR}_{u}\left(X / w_{t}\right), P_{t}-p_{t}\right\rangle & =\frac{1}{4}\left(\left\|\frac{\partial}{\partial w_{t}}\left(\operatorname{VaR}_{u}\left(X / w_{t}\right)\right)+\left(P_{t}-p_{t}\right)\right\|^{2}\right. \\
& \left.+\left\|\frac{\partial}{\partial w_{t}}\left(\operatorname{VaR}_{u}\left(X / w_{t}\right)\right)-\left(P_{t}-p_{t}\right)\right\|^{2}\right)
\end{aligned}
$$

Then, it follows that:

$$
\begin{aligned}
f_{w}^{-1}\left(w_{t}\right)\left\langle\frac{\partial}{\partial w_{t}}\left(\operatorname{VaR}\left(X / w_{t}\right)\right), P_{t}-p_{t}\right\rangle & =\frac{1}{4} \frac{\partial}{\partial w_{t}}\left(f_{w}^{-1}\left(w_{t}\right)\right)\left(\left\|\frac{\partial}{\partial w_{t}}\left(\operatorname{VaR}_{u}\left(X / w_{t}\right)\right)+\left(P_{t}-p_{t}\right)\right\|^{2}\right. \\
& \left.+\left\|\frac{\partial}{\partial w_{t}}\left(\operatorname{VaR}_{u}\left(X / w_{t}\right)\right)-\left(p_{t}-P_{t}\right)\right\|^{2}\right)
\end{aligned}
$$

hence the result : 


$$
\begin{aligned}
C\left(u_{1}, \ldots, u_{n} / w_{t}\right) & =\frac{1}{4} f_{w}^{-1}\left(w_{t}\right)\left(\left\|\frac{\partial}{\partial w_{t}}\left(\operatorname{VaR} R_{u}\left(X / w_{t}\right)\right)+\left(P_{t}-p_{t}\right)\right\|^{2}\right. \\
& \left.+\left\|\frac{\partial}{\partial w_{t}}\left(\operatorname{VaR}_{u}\left(X / w_{t}\right)\right)-\left(P_{t}-p_{t}\right)\right\|^{2}\right)
\end{aligned}
$$

\subsection{The CVaR in an Euclidean space}

The Tail-VaR (TVaR) is derivative coherent risk measure of the VaR. For a given confidence level $\alpha \in] 0,1[$, it follows that

$$
\operatorname{TVaR}_{\alpha}(X)=\frac{1}{1-\alpha} \int_{\alpha}^{1} \operatorname{VaR}_{\xi}(X) d \xi=\frac{1}{1-\alpha}\left[\mathbb{E}[X]-\int_{0}^{\alpha} \operatorname{VaR}(X) d \xi\right]
$$

The XTVaR is the average amount of ruins beyond the VaR;

$$
X T \operatorname{Va} R_{\alpha}(X)=T \operatorname{Va} R_{\alpha}(X)-\operatorname{VaR}_{\alpha}(X)
$$

the relation 21 we get the following proposition.

Proposition 5. The Tail-VaR (TVaR) is derivative coherent risk measure of the VaR. For a given confidence level $\alpha \in] 0,1[$, it follows that

$$
\begin{aligned}
C\left(u_{1}, \ldots, u_{n} / w_{t}\right) & =f_{w}^{-1}\left(w_{t}\right)\left\langle\frac{\partial}{\partial w_{t}}\left(T V a R_{u}\left(X / w_{t}\right)\right),\left(P_{t}-p_{t}\right)\right\rangle \\
& -f_{w}^{-1}\left(w_{t}\right)\left\langle\frac{\partial}{\partial w_{t}}\left(X T V a R_{u}\left(X / w_{t}\right)\right),\left(P_{t}-p_{t}\right)\right\rangle
\end{aligned}
$$

Furthermore

$$
C\left(u_{1}, \ldots, u_{n} / w_{t}\right)=f_{w}^{-1}\left(w_{t}\right) \frac{1}{2}\left(\left\|\frac{\partial}{\partial w_{t}}\left(\operatorname{TVa} R_{u}\left(X / w_{t}\right)\right)\right\|^{2}-\left\|\frac{\partial}{\partial w_{t}}\left(X T V a R_{u}\left(X / w_{t}\right)\right)\right\|^{2}\right)
$$

Proof. For relation (21) and the Proposition (3) we obtain the following equality :

$$
\begin{aligned}
C\left(u_{1}, \ldots, u_{n} / w_{t}\right) & =f_{w}^{-1}\left(w_{t}\right)\left[\left\langle\frac{\partial}{\partial w_{t}}\left(\operatorname{TVa} R_{u}\left(X / w_{t}\right)\right),\left(P_{t}-p_{t}\right)\right\rangle\right. \\
& \left.-\left\langle\frac{\partial}{\partial w_{t}}\left(X \operatorname{TVa} R_{u}\left(X / w_{t}\right)\right),\left(P_{t}-p_{t}\right)\right\rangle\right]
\end{aligned}
$$


Then, it follows that:

$$
\begin{aligned}
C\left(u_{1}, \ldots, u_{n} / w_{t}\right) & =f_{w}^{-1}\left(w_{t}\right) \frac{1}{4}\left[\left\|\frac{\partial}{\partial w_{t}}\left(T \operatorname{Va} R_{u}\left(X / w_{t}\right)\right)+\left(P_{t}-p_{t}\right)\right\|^{2}\right. \\
& \left.-\left\|\frac{\partial}{\partial w_{t}}\left(X T V a R_{u}\left(X / w_{t}\right)\right)+\left(P_{t}-p_{t}\right)\right\|^{2}\right] \\
& =f_{w}^{-1}\left(w_{t}\right) \frac{1}{4}\left[2\left(\left\|\frac{\partial}{\partial w_{t}}\left(T V a R_{u}\left(X / w_{t}\right)\right)\right\|^{2}+\left\|P_{t}-p_{t}\right\|^{2}\right)\right. \\
& \left.-2\left(\left\|X T V a R_{u}\left(X / w_{t}\right)\right\|^{2}+\left\|\left(p_{t}-P_{t}\right)\right\|^{2}\right)\right]
\end{aligned}
$$

it comes that :

$C\left(u_{1}, \ldots, u_{n} / w_{t}\right)=f_{w}^{-1}\left(w_{t}\right) \frac{1}{2}\left(\left\|\frac{\partial}{\partial w_{t}}\left(\operatorname{TVa} R_{u}\left(X / w_{t}\right)\right)\right\|^{2}-\left\|\frac{\partial}{\partial w_{t}}\left(X \operatorname{TVa} R_{u}\left(X / w_{t}\right)\right)\right\|^{2}\right)$.

\subsection{Performance Measures and the Distribution of $L \& P$ of a Portfolio}

Proposition 6. Let $w=\left(w_{1}, \ldots, w_{n}\right)^{T} \in \mathbb{R}^{n}$ a portfolio consisting of $n$ capital (the allocation of capital) and $S_{t}=\left(S_{1, t}, \ldots, S_{n, t}\right)^{T}$ the non-negative random vector representing the capital at the moment $t$. Then geometric yield

$$
\begin{aligned}
R_{t} & =\log \left(\frac{\left(\left[\left(\sigma\left(S_{t}\right)\right)^{2}+\left(\mathbb{E}\left(S_{t}\right)\right)^{2}\right]\right)^{1 / 2}}{\left(\left[\left(\sigma\left(S_{t-1}\right)\right)^{2}+\left(\mathbb{E}\left(S_{t-1}\right)\right)^{2}\right]\right)^{1 / 2}}\right. \\
& \left.+\frac{n \times \Delta_{t}}{\left(\left[(\sigma(w))^{2}+(\mathbb{E}(w))^{2}\right]\right)^{1 / 2} \times\left(\left[\left(\sigma\left(S_{t-1}\right)\right)^{2}+\left(\mathbb{E}\left(S_{t-1}\right)\right)^{2}\right]\right)^{1 / 2}}\right)
\end{aligned}
$$

where $\sigma(\cdot)$ is a standard deviation and $\mathbb{E}(\cdot)$ is a mean and with $\Delta_{t}$ all the interim payments obtained between the dates $t-1$ and $t$.

The distribution of $\left(P_{t+\tau}-P_{t}\right)$ is called profit distribution loss that expresses the change in the value of the portfolio.

Proof. The $P_{t}$ value of the portfolio is given by :

$$
P_{t}=\sum_{j=1}^{n} w_{j} S_{j, t}
$$


The profits and losses associated with holding the asset are then defined by the difference

$$
P \& L=P_{t}+\Delta_{t}-P_{t-1}=\sum_{j=1}^{n} w_{j}\left(S_{j, t}-S_{j, t-1}\right)+\Delta_{t} .
$$

The equality 26 becomes using the scalar product: :

$$
P \& L=\left\langle w, S_{t}-S_{t-1}\right\rangle=\|w\|\left\|S_{t}-S_{t-1}\right\|+\Delta_{t}
$$

Indeed on the same types of considerations of the section 3.1 ( $w$ and $S_{t}$ are linked). Then

$$
P \& L=\left(\sum_{j=1}^{n} w_{j}^{2}\right)^{1 / 2} \times\left(\sum_{j=1}^{n}\left(S_{j, t}-S_{j, t-1}\right)^{2}\right)^{1 / 2}+\Delta_{t}
$$

These losses and profits are expressed in the form of a geometric return noted $R_{t}$ :

$$
R_{t}=\log \left(\frac{P_{t}+\Delta_{t}}{P_{t-1}}\right)=\log \left(\frac{\left(\sum_{j=1}^{n} w_{j}^{2}\right)^{1 / 2} \times\left(\sum_{j=1}^{n} S_{j, t}^{2}\right)^{1 / 2}+\Delta_{t}}{\left(\sum_{j=1}^{n} w_{j}^{2}\right)^{1 / 2} \times\left(\sum_{j=1}^{n} S_{j, t-1}^{2}\right)^{1 / 2}}\right)
$$

if we use the following relation;

$$
\mathbb{E}\left(S_{t}^{2}\right)=\left[\frac{1}{n}\left(\sigma\left(S_{t}\right)\right)^{2}+\left(\mathbb{E}\left(S_{t}\right)\right)^{2}\right]^{1 / 2} .
$$

It comes that:

$$
\begin{aligned}
R_{t} & =\log \left(\frac{\left(\left[\left(\sigma\left(S_{t}\right)\right)^{2}+\left(\mathbb{E}\left(S_{t}\right)\right)^{2}\right]\right)^{1 / 2}}{\left(\left[\left(\sigma\left(S_{t-1}\right)\right)^{2}+\left(\mathbb{E}\left(S_{t-1}\right)\right)^{2}\right]\right)^{1 / 2}}\right. \\
& \left.+\frac{n \times \Delta_{t}}{\left(\left[(\sigma(w))^{2}+(\mathbb{E}(w))^{2}\right]\right)^{1 / 2} \times\left(\left[\left(\sigma\left(S_{t-1}\right)\right)^{2}+\left(\mathbb{E}\left(S_{t-1}\right)\right)^{2}\right]\right)^{1 / 2}}\right)
\end{aligned}
$$

It is assumed that the return on the date $t$, i.e. $R_{t}$, is a real random variable.

Corollary 1. Let $w=\left(w_{1}, \ldots, w_{n}\right)^{T} \in \mathbb{R}^{n}$ a portfolio consisting of $n$ capital (the allocation of capital) and $S_{t}=\left(S_{1, t}, \ldots, S_{n, t}\right)^{T}$ the non-negative random vector representing the capital at the moment $t$. Then geometric yield

$$
R_{t} \simeq \frac{n \times \Delta_{t}}{\left(\left[(\sigma(w))^{2}+(\mathbb{E}(w))^{2}\right]\right)^{1 / 2} \times\left(\left[\left(\sigma\left(S_{t}\right)\right)^{2}+\left(\mathbb{E}\left(S_{t}\right)\right)^{2}\right]\right)^{1 / 2}}
$$

where $\sigma(\cdot)$ is a standard deviation and $\mathbb{E}(\cdot)$ is a mean and with $\Delta_{t}$ all the interim payments obtained between the dates $t-1$ and $t$. 
Proof. By taking the relationship (24) and as $S_{t}$ and $S_{t-1}$ are of the same nature we have :

$$
\sigma\left(S_{t}\right) \simeq \sigma\left(S_{t-1}\right) \text { and } \mathbb{E}\left(S_{t}\right) \simeq \mathbb{E}\left(S_{t-1}\right) .
$$

and view the limited developmental formula in the neighborhood of zero $\log (1+x) \simeq x$ it comes that

$$
R_{t}=\log \left(1+\frac{n \times \Delta_{t}}{\left(\left[(\sigma(w))^{2}+(\mathbb{E}(w))^{2}\right]\right)^{1 / 2} \times\left(\left[\left(\sigma\left(S_{t}\right)\right)^{2}+\left(\mathbb{E}\left(S_{t}\right)\right)^{2}\right]\right)^{1 / 2}}\right)
$$

Hence the result.

\subsection{Conclusion and Discussion}

We thought to introduce the notion of product in the stochastic modeling of the copula and the value at risk. Because it allows us to combine precisely these two notions (Copula, $\mathrm{VaR}$ ). One of the characteristics of the scalar product is the fact that it makes it possible to move the diferential from one component to another. The different relations that we obtained in this document allow to establish a close link between copula and the value at risk through an analytical expression with the norms. It becomes quite easy to calculate the values of VaR when we know the value of the copula and the type of copula.

\section{References}

[1] Artzner, P., Delbaen, F., Eber, J.-M., and Heath, D. (1999). "Coherent measures of risk." Mathematical Finance, 9 (3): 203-228.

[2] Cardin M., (2010) Some classes of multivariate risk measures,Springer pp 63-730 Doi: 10.1007/978-88-470-1481-7_7

[3] Cousin A., Di Bernardino E. (2012) A multivariate extension of Value-at-Risk and Conditional- Tail-Expectation

[4] Basel II, 2006- Basel Committee on Banking Supervision, International Convergence of Capital Measurement and Capital Standards. Basel, June 2006, http://www.bis.org.

[5] Bouy E.(2002) Multivariate Extremes at Work for Portfolio Risk Measurement. Warwick Business School Working Paper Series, WP01-10.

[6] Charpentier, A. and Segers, J. (2009). Tails of multivariate Archimedean copulas. J. Multivariate Anal. 100, 1521-1537. MathSciNet: MR2514145 doi:10.1016/j.jmva.2008.12.015 
[7] Joe, H. (1997). Multivariate Models and Dependence Concepts - Monographs on Statistics and Applied Probabilty 73, Chapman and Hall, London.

[8] Patton, A. J. (2001), Modelling Time-Varying Exchange Rate Dependence Using the Conditional Copula, PhD thesis, Department of Economics, UCSD.

[9] Nelsen, R. B. (1999). An Introduction to Copulas. Springer, New York.

[10] Patton, A.J., (2006), Modelling Asymmetric Exchange Rate Dependence, International Economic Review, 47(2), 527-556.

[11] Basel Committee on Banking Supervision, International Convergence of Capital Measurement and Capital Standards, Basel, June 2006, www.bis.org.

[12] Soustra, F. (2006), ' Pricing of synthetic cdo tranches, analysis of base correlation and an intro- duction to dynamic copulas', M emoire de maitrise, HEC Montr eal .

[13] Embrechts, P. Mikosch, T. Klppelberg, C. (1997). "Modelling Extremal Events: for Insurance and Finance." Springer-Verlag, London.

[14] Embrechts, P., A. McNeil and D. Straumann (2002), Correlation and Dependence in Risk Man- agement: Properties and Pitfalls, in M. Dempster (ed.), Risk Management:Value at Risk and Beyond, Cambridge University Press, pp. 176-223.

[15] Rockafeller RT (2007), Coherent approaches to risk in optimization under uncertainty. Tutorials in Operations Research INFORMS, 38-61.

[16] Rootzn, H. and Klppelberg C. (1999). "A Single Number Can't Hedge Against Economic Catastrophes" Working Paper, Munich University of Technology.

[17] Nelsen, R.B. (1999). An Introduction to copulas- Lectures notes in Statistics 139, Springer-Verlag (ISBN 10: 0387986235 / ISBN 13: 9780387986234 )

[18] Basel Committee on Banking Supervision, 2010. Basel III: A Global Regulatory Framework for More Resilient Banks and Banking Systems. http://www.bis.org/publ/bcbs189_dec2010.pdf, December.

[19] Beirlant, J., Goegebeur, Y., Segers, J., and Teugels, J. (2005). Statistics of Extremes: theory and application -Wiley, Chichester, England.

[23] Carreau J. (2004). Estimation de densit conditionnelle lorsque l'hyppothse de normalit est insatisfaisante - thse de doctorat.

[21] Clauss, P. (2008). Thorie des copules - Elments de cours sur les copules multivariesENSAI.

[22] Yves Bernadin Vini Loyara, Remi Guillaume Bagr and Diakarya Barro. (2017). Multivariate risk modeling for financial portfolio management and climate application. Far East Journal of Mathematical Sciences (FJMS). 
[23] Karl Friedrich Siburg, Pavel A. Stoimenov. (2007) . A scalar product for copulas. Journal of mathematical analysis and Application. 